\title{
Zero-lag Long Range Synchronization of Neurons Is Enhanced by Dynamical Relaying
}

\author{
Raul Vicente ${ }^{1,2}$, Gordon Pipa ${ }^{1,2}$, Ingo Fischer ${ }^{3}$ and Claudio R. Mirasso ${ }^{4}$ \\ 1 Max-Planck Institute for Brain Research, Deutschordenstrasse 46, \\ 60528 Frankfurt am Main, Germany \\ ${ }^{2}$ Frankfurt Institute for Advanced Studies, Max-von-Laue Strasse 1, \\ 60438 Frankfurt am Main, Germany \\ 3 Dept. of Applied Physics and Photonics (TONA) Vrije Universiteit Brussel, \\ Pleinlaan 2, B-1050 Brussel, Belgium \\ ${ }^{4}$ Dept. de Fisica Universitat de les Illes Balears, Crta de Valldemossa km 7.5, \\ E-07071 Palma de Mallorca, Spain
}

\begin{abstract}
How can two distant neural assemblies synchronize their firings at zero-lag even in the presence of non-negligible delays in the transfer of information between them? Here we propose a simple network module that naturally accounts for zero-lag neural synchronization for a wide range of temporal delays. In particular, we demonstrate that isochronous (without lag) millisecond precise synchronization between two distant neurons or neural populations can be achieved by relaying their dynamics via a third mediating single neuron or population.
\end{abstract}

\section{Introduction}

Neural synchronization stands today as one of the most promising mechanisms to counterbalance the huge anatomical and functional specialization of the different brain areas [1-3]. In particular, it proposes the formation of transiently synchronized neural assemblies during few hundreds of milliseconds as the underlying process to bind several local neural dynamics. Consequently, neural synchrony can provide a dynamic and reconfigurable mechanism for large-scale integration of distributed brain activity and serve as an efficient code to complement the rate modulation and overcome some of its limitations. The synchrony hypothesis has been supported by experimental findings demonstrating that millisecond precise synchrony of neuronal oscillations across well separated cortical areas plays an essential role in visual coherent perception and other high-cognitive tasks $[3,4]$.

However, and albeit more evidence is being accumulated in favor of its functional role as a binding mechanism of distributed neural responses, the physical and anatomical substrate for such a dynamic and precise synchrony, especially zero-lag even in the presence of non-negligible delays, remains unclear [4]. Several mechanisms have been proposed to explain the appearance of synchronization between neural populations. Inhibitory connections and gap junctions have been proposed to increase the stability of the synchronous state [5]. In general, these 
and other approaches require either the precise tuning of properties such as the synaptic rise time or rely on rather complex architectures [6] while exhibiting several limitations in the range of synchronization attainable.

In addition, the problem of the communication delays between the neural units involved in the interaction, although fundamental, has been hardly faced [7]. Conduction and synaptic delays between the brain areas which are observed to synchronize can amount to several tens of milliseconds. How under such latency times the reciprocal interactions between two brain regions can lead the associated neural populations come into sync without almost any lag?

Here we propose a simple network motif that is able to naturally lead to the zero-lag synchronization between two arbitrarily distant neural populations. The basic idea is that when two spiking neurons interact not directly but through a third mediating neuronal unit, the redistribution of the dynamics performed by this central unit leads in a robust and self-consistent manner toward the zero-lag synchronization of the outer neurons [8]. This simple network module is expected to exist within the complex functional architecture of the brain and especially within the reciprocal thalamocortical interactions. It is significant that recent studies have demonstrated the constant latency between the thalamus (the main relay unit of sensory information in the brain) and almost any area in the mammalian neocortex [9]. Remarkably, this occurs irrespective of the very different distances that separate the thalamic nuclei and the cortex regions involved. This means that an action potential generated in a thalamic cell will take the same time to reach a cortical neuron independently of the thalamocortical afferent used to propagate the spike. To our purposes, this implies that a thalamocortical circuit is an ideal representation of this network module. With the proposed network motif the synchronous state can be achieved after the exchange of a few spikes and consequently within a few tens of milliseconds. This time scale is perfectly compatible with the required processing times of information found experimentally [4].

\section{Methods}

In order to test the synchronization properties of such neural circuits we simulated the dynamics of Hodgkin-Huxley $(\mathrm{HH})$ neurons that interact with each other via reciprocal synaptic connections with an intermediate third neuron of the same type.

\subsection{Mathematical Model}

The dynamics of the membrane potential of each neuron is modeled by the classical Hodgkin-Huxley equations [10] with the addition of appropriate synaptic currents to mimic the chemical coupling between neurons. 
The temporal evolution of the voltage across the membrane is given by

$$
C \frac{d V}{d t}=-g_{N a} m^{3} h\left(V-E_{N a}\right)-g_{K} n^{4}\left(V-E_{k}\right)-g_{L}\left(V-E_{L}\right)+I_{e x t}+I_{s y n},
$$

where $C=1 \mu \mathrm{F} / \mathrm{cm}^{2}$ is the membrane capacitance, the constants $g_{N a}=120$ $\mathrm{mS} / \mathrm{cm}^{2}, g_{K}=36 \mathrm{mS} / \mathrm{cm}^{2}$, and $g_{L}=0.3 \mathrm{mS} / \mathrm{cm}^{2}$ are the maximal conductances of the sodium, potassium, and leakage channels, and $E_{N a}=50 \mathrm{mV}, E_{K}=$ $-77 \mathrm{mV}$, and $E_{L}=-54.5 \mathrm{mV}$ stand for the corresponding reversal potentials. According to Hodgkin and Huxley formulation the voltage-gated ion channels are described by the following set of differential equations

$$
\begin{gathered}
\frac{d m}{d t}=\alpha_{m}(V)(1-m)-\beta_{m}(V) m, \\
\frac{d h}{d t}=\alpha_{h}(V)(1-h)-\beta_{h}(V) h, \\
\frac{d n}{d t}=\alpha_{n}(V)(1-n)-\beta_{n}(V) n,
\end{gathered}
$$

where the gating variables $m(t), h(t)$, and $n(t)$ represent the activation and inactivation of the sodium channels and the activation of the potassium channels, respectively. The experimentally fitted voltage-dependent transition rates are

$$
\begin{gathered}
\alpha_{m}(V)=\frac{0.1(V+40)}{1-\exp (-(V+40) / 10)}, \\
\beta_{m}(V)=4 \exp (-(V+65) / 18), \\
\alpha_{h}(V)=0.07 \exp (-(V+65) / 20), \\
\beta_{h}(V)=[1+\exp (-(V+35) / 10)]^{-1}, \\
\alpha_{n}(V)=\frac{(V+55) / 10}{1-\exp (-0.1(V+55))}, \\
\beta_{n}(V)=0.125 \exp (-(V+65) / 80) .
\end{gathered}
$$

The synaptic transmission between neurons is modeled by a postsynaptic conductance change with the form of an alpha-function

$$
\alpha(t)=\frac{1}{\tau_{d}-\tau_{r}}\left(\exp \left(-t / \tau_{d}\right)-\exp \left(-t / \tau_{r}\right)\right)
$$

where the parameters $\tau_{d}$ and $\tau_{r}$ stand for the decay and rise time of the function and determine the duration of the response. Consequently, the the synaptic current takes the form

$$
I_{\text {syn }}(t)=-g_{\max } \sum_{\tau_{l}} \sum_{\text {spikes }} \alpha\left(t-t_{\text {spike }}-\tau_{l}\right)\left(V(t)-E_{\text {syn }}\right),
$$


where $g_{\max }$ describes the maximal synaptic conductance and the internal sum is extended over the train of presynaptic spikes occurring at $t_{\text {spike }}$. The delays arising from the finite conduction velocity of axons are taken into account through the latency time $\tau_{l}$ in the alpha-function. Thus, the external sum covers the different latencies that arise from the existence of multiple synaptic connections between two different neural populations. Excitatory and inhibitory transmissions are differentiated by setting the synaptic reversal potential to be $E_{\text {syn }}=0$ $\mathrm{mV}$ or $E_{\text {syn }}=-80 \mathrm{mV}$, respectively.

Finally, the external current $I_{\text {ext }}$ stimulation is adjusted to a constant value of $10 \mu \mathrm{A} / \mathrm{cm}^{2}$. Under such conditions a single Hodgkin-Huxley type neuron enters into a regime of periodic firing with a natural period of $T_{n a t}=14.66 \mathrm{~ms}$. When inspecting the role of noise, white Gaussian stochastic fluctuations were added to the otherwise constant level of the external current.

\subsection{Geometry}

We consider a neural circuit composed by two Hodgkin-Huxley neurons interacting with a relay neuron of the same class. This mediating neuron is receiving input from the two outer neurons and projecting output toward them. Latency times are included in the interaction to capture the non-negligible conduction delays occurring in the transmission of spikes. This neural circuit can be thought as a simple model of two neuronal populations $(\alpha$ and $\beta$ ) interacting through a set of neurons whose dynamics is summarized by a single mediating population $c$ with a given transfer function (see Fig. 1).

\section{A}

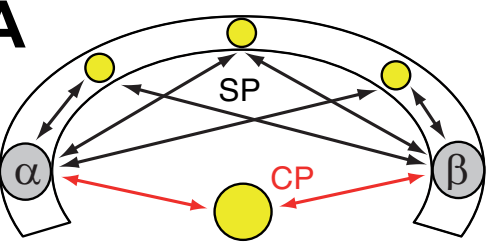

SP: Set of paths CP: Central path
B
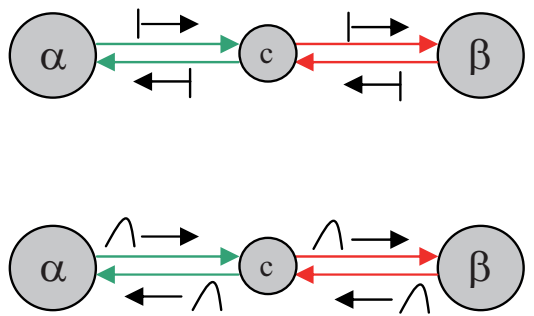

Fig. 1. a) Sketch of a neural circuit with recurrent connections mapped to the interaction between three neural populations $(\alpha, \beta$, and a central population $c$.) b) Interaction between populations $\alpha$ and $\beta$ through the mediating element $c$ with single (top) or distributed (bottom) synaptic delays.

Individual temporal delays of the arrival of presynaptic potentials (i.e., latency times) were modelled by a gamma distribution to mimic the multiple con- 
nections between the neural populations involved in the synchronization process.

The probability density function of the distribution of delays is then

$$
f\left(\tau_{l}\right)=\tau_{l}^{k-1} \frac{\exp \left(-\tau_{l} / \theta\right)}{\theta^{k} \Gamma(k)}
$$

where $k$ and $\theta$ are shape and scale parameters of the gamma distribution. The mean time delay is given by $\hat{\tau}_{l}=k \theta$.

\subsection{Numerical Integration}

The set of equations (1-12) is numerically integrated using the Heun method with a time step of $0.02 \mathrm{~ms}$.

Starting from random initial conditions each neuron is first simulated without any synaptic coupling for $200 \mathrm{~ms}$ after which frequency adaptation has occurred and each neuron settles into a periodic firing regime with a well defined frequency. The relation between the phases of the oscillatory activities of the neurons at the end of this heating time was entirely determined by the initial conditions. Following this period and once the synaptic transmission has been activated, a simulation time of 3 seconds is recorded. This allowed us to trace the change in the relative timing of the spikes induced by the synaptic coupling in this neural circuit.

\subsection{Data Analysis}

The strength of the synchronization and the phase-difference between each individual pair of neurons $(m, n)$ were derived by the computation of the order parameter defined as

$$
\rho(t)=\frac{1}{2}\left|\exp \left(i \phi_{m}(t)\right)+\exp \left(i \phi_{n}(t)\right)\right|,
$$

which takes the value of 1 when two oscillators are moving in-phase and 0 in an anti-phase regime. In order to compute this quantifier it is only necessary to estimate the phases of the individual neural oscillators. An advantage of this method is that one can easily reconstruct the phase of a neuronal oscillation from the train of spikes without the need of recording the full membrane potential time series [11]. The idea behind is that the time interval between two well defined events (such as action potentials) define a complete cycle and the phase increase during this time amounts to $2 \pi$. Then, linear interpolation is used to assign a value to the phase during the spike events.

Cross-correlation analysis of the membrane potentials was also computed in order to check the results obtained from the order parameter estimation. 


\section{Results}

Prior to the illustration of the results obtained from the direct simulation of equations (1-12), we first characterize in Fig. 2 the response of a single HodgkinHuxley neuron to the arrival of a presynaptic potential and the subsequent change in the postsynaptic conductivity. In particular, we computed how much the period of the oscillation of a single $\mathrm{HH}$ neuron is changed as a function of the phase at which this single perturbation is received. This phase response curve (PRC) contains useful information about the synchronization properties of the oscillators involved.
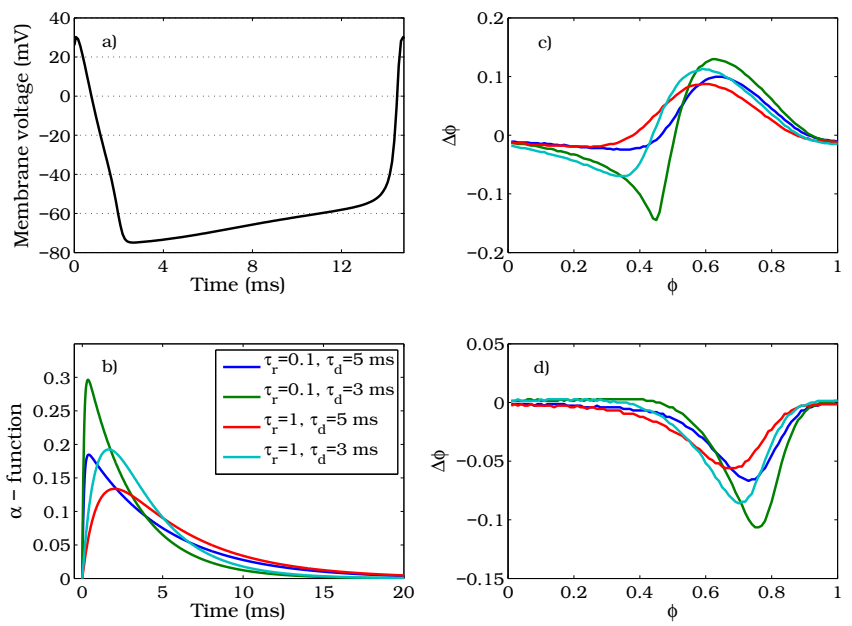

Fig. 2. a) Membrane voltage of an unperturbed HH neuron during a cycle of oscillation. b) Alpha-functions with different rise and decay times indicated in the legend. c) PRCs for excitatory synapses $\left(E_{\text {syn }}=0 \mathrm{mV}\right)$. d) PRCs for inhibitory synapses $\left(E_{\text {syn }}=-80 \mathrm{mV}\right)$. The PRC is defined as $\Delta \phi=1-T_{p e r} / T_{n a t}$, where $T_{p e r}$ stands for the length of the cycle containing the perturbation. $\phi$ represents the phase at which the perturbation is received. The colors in panels c) and d) code for alpha-functions of the same color shown in b). $g_{\max }=0.2$.

Figure 2 shows the trace of the voltage potential of an unperturbed $\mathrm{HH}$ neuron and the phase response curves to alpha-functions with different synaptic rise and decay times. The Hodgkin and Huxley neuron is known to produce a response such that a perturbation can advance or retard the next spike as it is seen in panel c) where an excitatory coupling was simulated. However, it is observed in panel d) that in the investigated regime, an inhibitory synapse can only retard the firing of the next action potential. It is also noticed that due to the finite duration of the alpha-function the value of the PRCs for excitatory 
synapses differs from zero in the neighborhood of a spike $(\phi=0,1)$.

The different synchronization characteristics of inhibitory and excitatory synapses in two-coupled neuron models and the role of the synaptic rise and decay times can be attributed to the different features of their respective PRCs $[5,12]$. Nevertheless, here we propose a network module that naturally provides a robust mechanism for synchronizing two neural populations with zero-phase lag. This effect is based on the dynamical relaying provided by a third population, and opposite to directly-coupled neuron models it turns out to be largely independent of the characteristics of the synaptic transmission. Figure 3 displays the time traces of the membrane potential for the three neurons coupled as in the module of Fig.1 with excitatory synapses.

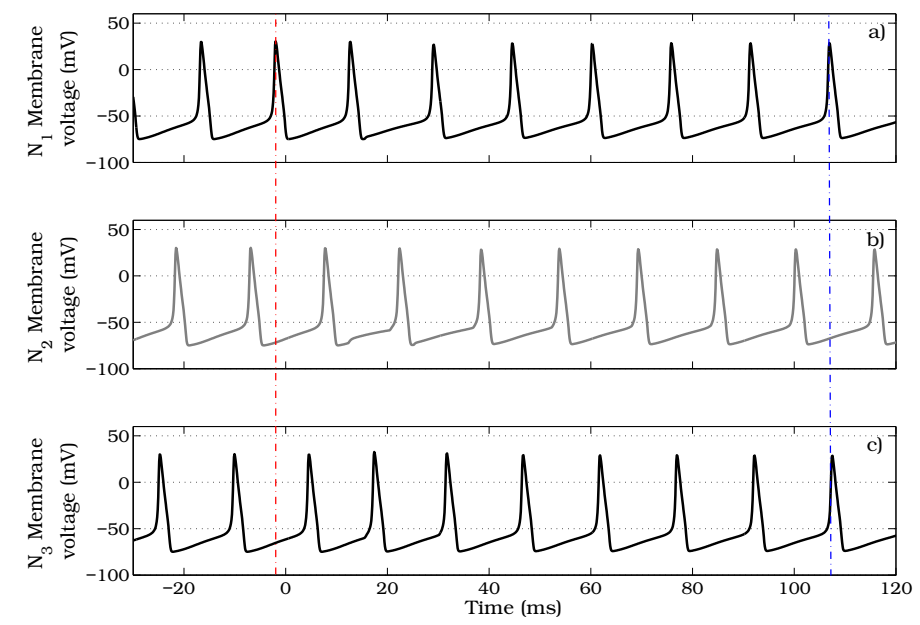

Fig. 3. a), b), and c) show the evolution of membrane potential of the three neurons interacting through the network sketched in Fig.1. Neuron 2 is the relay neuron. The synaptic coupling is activated at $t=0 \mathrm{~ms}$ with a $g_{\max }=0.5$. The gamma distribution of the latency times is chosen to be at the limit at which it tends to a delta distribution centered in $8 \mathrm{~ms}$. The alpha-function used for the synaptic coupling had a rise time of $\tau_{r}=0.1 \mathrm{~ms}$ and a decay time of $3 \mathrm{~ms}$.

It is observed that once the synaptic coupling is activated the network module consistently self-organizes toward the state in which the outer neurons synchronize their spikes. The zero-lag synchronization arises as a consequence of the relay and redistribution of excitatory postsynaptic potentials (EPSP) performed by the central neuron. The EPSPs induced in such a neural circuit are responsible to slightly modify the firing timing of the postsynaptic cells in a manner that the outer neurons tend to fire at unison after a few spikes irrespective of 
the initial conditions. We have checked that this phenomenon also occurs for inhibitory synapses and different synaptic temporal scales.

To inspect if the unavoidable noise sources around a neuron are able to disrupt the synchronization we simulated the dynamics of the HH neurons with independent additive white noise in the external current input of each cell. Figure 4 shows that even in the presence of moderate noise intensities the synchronization process takes place.

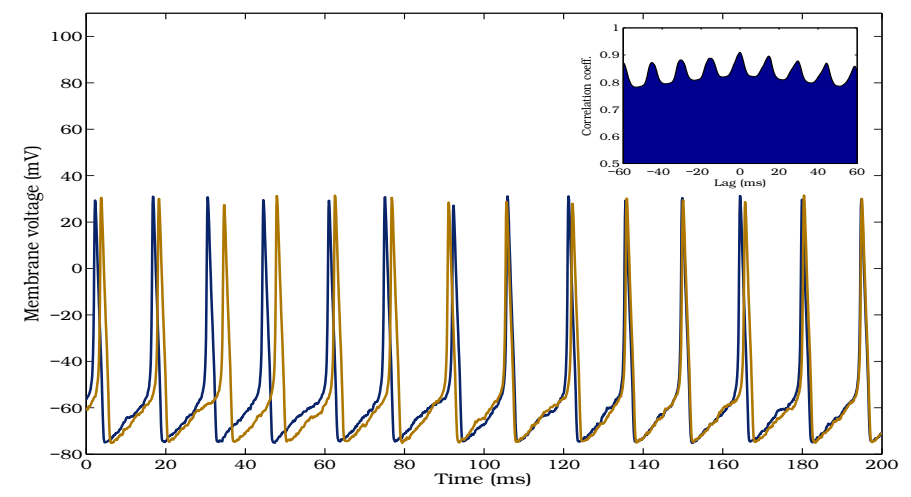

Fig. 4. The large panel shows the temporal evolution of the outer neurons in the presence of noise once synaptic coupling is activated at $t=0 \mathrm{~ms}$. The noise level was adjusted to $\sigma=1 \mu \mathrm{A} \mathrm{ms} s^{1 / 2} / \mathrm{cm}^{2}$. It is clear that even the spikes become more irregular a good synchronization level is obtained at zero-lag. The inset displays the cross-correlation function of the voltage temporal series for a 3 second simulation.

At this point, a crucial question is whether this synchronization transition is particular to single latency synaptic pathways or it is maintained for broad distributions of conduction delays. To answer such issue we have represented in Figure 5 the order parameter of the neuronal oscillations of the outer cells for several delay distributions. In particular, we scanned the shape factor and the mean value of the distribution of delays. The results indicate the existence of a broad region of delays (between $2 \mathrm{~ms}$ and $9 \mathrm{~ms}$ ) where for almost any shape factor the outer neurons come into sync. Only distributions with an unrealistic small shape factor (nearly decaying exponentials distributions) are unable to produce synchrony regardless the average delay of the synaptic connections. The drop in the synchronization quality found around $\hat{\tau}_{l} \sim 10 \mathrm{~ms}$ is associated to an irregular firing state of the neurons where no locking behavior is found.

Thus, zero-phase synchronization can take place in such network module even in the presence of broad distributions in the delays communicating two neural populations. Similar behaviors are found for inhibitory synapses and 


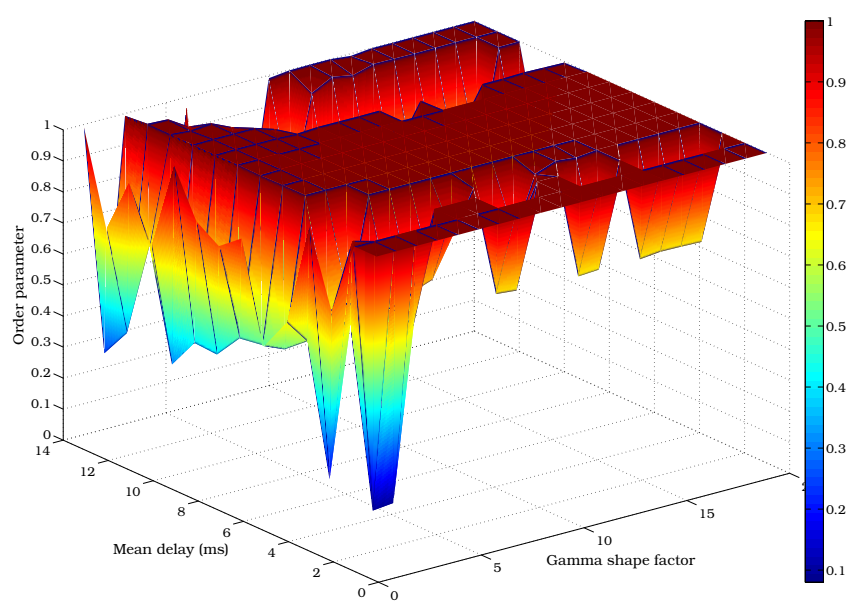

Fig. 5. Order parameter of the phases of the outer neurons as a function of the shape factor and mean delay of the gamma distribution of delays. $g_{\max }=0.2, E_{\text {syn }}=0 \mathrm{mV}$. The alpha-function used for the synaptic coupling had a rise time of $\tau_{r}=0.1 \mathrm{~ms}$ and a decay time of $3 \mathrm{~ms}$.

alpha-functions with different durations demonstrating that the synchronization process in such a network is largely independent of the particular characteristics of the PRCs of the neuron.

Up to now, only symmetrical distributions of delays have been considered in the pathways from the relay neuron to each one of the outer units. However, when different distributions are simulated for each of the pathways we have found that the phase lag between the outer neurons usually deviates from zero with the cell with the shortest mean distance to the relay unit leading the dynamics. Nevertheless, it is quite remarkable that broad distributions (large shape factors) allow for almost zero-lag synchronization even in the presence of differences of several milliseconds in the average delay of both pathways.

\section{Discussion}

We have introduced a simple and extremely robust network motif that is able to account for the zero-phase synchronization of distant neural elements in a natural way. This robust synchronization arises as a consequence of the relay and redistribution of the dynamics performed by a mediating neuron. As a consequence and in opposition to previous works, neither inhibitory, gap junctions, nor complex networks need to be invoked to provide a stable mechanism of zero-phase correlated activity of neural populations even in the presence of large 
conduction delays.

As a future direction we are working to provide a more physiologically detailed model of the network motif here presented in order to compare with experimental data. In particular, for the relay or thalamic neuron we are including a description of additional ionic channels (transient $\mathrm{Ca}^{2+}$ and $\mathrm{K}^{+}$as well as a persistent $\mathrm{Na}^{+}$conductance). Neural population modeling for each of the three involved pools of neurons is also under current research.

\section{Acknowledgments}

Research supported by the GABA project (European Commission, FP6-NEST contract 043309).

\section{References}

1. Von der Malsburg, C., Schneider, W.: A neural cocktail-party processor. Biological Cybernetics 54 (1986) 29-40

2. Rieke, F., Warland, D., De Ruyter van Steveninck, R.: Spikes: Exploring the Neural Code. MIT Press, Cambridge (1997)

3. Varela, F.J., Lachaux, J.P., Rodriguez, E., Martinerie, J.: The brainweb: phase synchronization and large-scale integration. Nature Reviews Neuroscience 2 (2001) 229-239

4. Singer, W.: Neuronal Synchrony: A Versatile Code for the Definition of Relations? Neuron 24 (1999) 49-65

5. Van Vreeswijk, C., Abbott, L.F., Ermentrout, B.: When inhibition not excitation synchronizes neural firing. Journal of Computational Neuroscience 1 (1994) 313-321

6. Lago-Fernandez, L.F., Huerta, R., Corbacho, F., Siguenza, J.A.: Fast response and temporal coherent oscillations in small-world networks. Physical Review Letters $\mathbf{8 4}$ (2000) 2758-2761

7. Freeman, W.: Characteristics of the synchronization of brain activity imposed by finite conduction velocity of axons. International Journal of Bifurcation and Chaos 10 (2000) 2307-2322

8. Fischer, I., Vicente, R., Buldu, J.M., Peil, M., Mirasso, C.R., Torrent, M.C., GarciaOjalvo, J.: Zero-lag synchronization via dynamical relaying. Physical Review Letters 97 (2006) 123902(1)-123902(4)

9. Salami, M., Itami, C., Tsumoto, T., Kimura, F.: Change of conduction velocity by regional myelination yields to constant latency irrespective of distance between thalamus and cortex. PNAS 100 (2003) 6174-6179

10. Hodgkin, A.L., Huxley, A.F.: A quantitative description of the membrane current and its application to conduction and excitation in nerve. Journal of Physiology 117 (1952) 500-544

11. Pikovsky, A., Rosenblum, M., Kurths, J.: Synchronization: A universal concept in nonlinear sciences. Cambridge University Press (2001)

12. Goel, P., Ermentrout, B.: Synchrony, stability and firing pattern is pulse-coupled oscillators. Physica D 163 (2002) 191-216 